\title{
Changes in Emotional Regulation May Underlie Emotional Changes in Early Parkinson's Disease Progression: A Multimodal Study.
}

\author{
Allison Eriksson ${ }^{2}$, Panagiota Tsitsi ${ }^{2,3}$, Mikkel C. Vinding ${ }^{2}$, Martin Ingvar ${ }^{2}$, Per \\ Svenningsson ${ }^{1,3}$, and Daniel Lundqvist ${ }^{2}$
}

1. Centrum for Neurology, Academic Specialist centrum, Region Stockholm, Sweden

2. Department of Clinical Neuroscience, Neuro, Karolinska Institutet, Stockholm, Sweden 3. Department of Neurology, Karolinska University Hospital, Stockholm, Sweden

\begin{abstract}
Author Note
We have no known conflict of interest to disclose. Correspondence concerning this article should be addressed to Allison Eriksson, Karolinska Institute, Department of Clinical Neuroscience, Nobels väg 9, Stockholm, Sweden, 171 77. Email: allison.eriksson@ki.se
\end{abstract}




\begin{abstract}
Parkinson's Disease (PD) is a neurodegenerative disorder which can substantially affect nonmotor functions related to emotional processing. However, previous studies investigating effects of PD on emotional processing have produced conflicting results. In the current study, we aimed to examine the underlying differences in emotional processing in PD by comparing how early-stage PD patients rate and react to emotional stimuli in three modalities on an Emotion Survey. Data analysis focused on identifying differences in emotion recognition, bias, and emotional range together with clinical outcome measures. Our results showed that PD patients were more accurate than healthy controls at identifying correct emotions. Furthermore, when clinical scores were correlated with ratings of emotional stimuli, PD patients alone showed a general increase in ratings and reactions to both positive and negative stimuli, thereby yielding significant correlations between clinical outcomes and emotional range in the PD patient group. Our results suggest that alterations in emotional regulation may underlie changes in emotional processing in early PD.
\end{abstract}

Keywords: Parkinson's Disease, emotion, multi-modal, ratings, range 


\section{Changes in Emotional Regulation May Underlie Emotional Changes in Early Parkinson's Disease Progression: A Multimodal Study.}

Parkinson's disease (PD) is a progressive neurodegenerative disorder characterized by a loss of dopaminergic neurons in the substantia nigra to putamen (Kalia \& Lang, 2015). The pathological damage related to this process gives rise to the motor symptoms typically associated with the disorder, such as bradykinesia, rigidity, and rest tremor, which serves as the basis of a clinical PD diagnosis (Postuma et al., 2015). Substantial nonmotor functions that are compromised in this disease could contribute to a lower quality of life for PD patients (Schapira, Chaudhuri, \& Jenner, 2017). These include autonomic dysfunction, cognitive impairment, and alterations in sleeping patterns (De Risi et al., 2018; Kalia \& Lang, 2015). Furthermore, neuropsychiatric disturbances, such as anxiety, and emotion dysregulation are prevalent as well, with up to $40 \%$ of PD patients reported to suffer from depression (Aarsland, Påhlhagen, Ballard, Ehrt, \& Svenningsson, 2011; Schapira et al., 2017).

Previous research on emotion processing in PD indicates that responses to emotional stimuli are biased, though results have often been conflicting (Gray \& Tickle-Degnen, 2010; Lundqvist, Svärd, Michelgård Palmquist, Fischer, \& Svenningsson, 2017). Some studies have found general evidence of emotional deficits in PD patients compared to healthy controls (HC), while others have found no significant differences between the groups (Gray \& Tickle-Degnen, 2010; Hälbig et al., 2011; Ille et al., 2016; Péron, Dondaine, Le Jeune, Grandjean, \& Vérin, 2012; Wabnegger et al., 2015). Research investigating specific emotion recognition using facial emotional stimuli has found impairments in recognition of negative basic emotions such as anger, fear, disgust, and sadness (Dujardin et al., 2004; Kan, Kawamura, Hasegawa, Mochizuki, \& Nakamura, 2002; Yip, Lee, Ho, Tsang, \& Li, 2003), while other studies have 
found comparable results for both PD patients and HC. (Blonder et al., 2013; Pell \& Leonard, 2005; Wabnegger et al., 2015). Studies of emotion recognition using vocal emotional stimuli have indicated that PD patients experience increased difficulties in distinguishing between different emotions when listening to voices (Breitenstein, Van Lancker, Daum, \& Waters, 2001; Péron et al., 2012). Breitenstein et al., (2001) found evidence of impaired recognition of vocal emotions in PD patients, and two subsequent meta-analyses suggest that PD patients show a greater deficiency in emotion recognition related to vocal stimuli than when presented with recognition of facial stimuli (Gray \& Tickle-Degnen, 2010; Péron et al., 2012).

Furthermore, the literature indicates that subjective appraisal of emotional intensity may also be altered in PD patients. Several studies have shown that PD patients exhibit negativity bias, or a stronger emotional response to negative emotional stimuli (De Risi et al., 2018; Hälbig et al., 2011; Lundqvist et al., 2017). In a study by Lundqvist and colleagues (2017), for example, PD patients displayed a negativity bias in the form of more negative valence ratings for disgusted, fearful, and sad faces. These patients displayed also a significantly larger emotional range in their responses, both in terms of emotional arousal and emotional valence.

So far, studies on emotional impairment in PD have primarily investigated emotion recognition using discrimination tasks (Péron et al., 2012). Moreover, most of these studies have been disproportionately focused on negative emotions, or include significantly fewer measures of positive emotion in comparison to negative emotions (Gray \& Tickle-Degnen, 2010; Péron et al., 2012). Studies which have included positive emotion categories, and analysis for emotional range, have shown a more general increase in emotional range so that negative stimuli are rated as more strongly negative, and positive stimuli more strongly positive in PD patients compared to HC (Lundqvist et al., 2017). Hence, the common tendency to rely 
primarily on negative stimuli could potentially contribute to the false appearance of a negativity bias among PD patients. More studies are required to confirm a negativity bias and to explore the possibility that a balanced inclusion of both positive and negative stimuli could give a wider and more nuanced view on changes in emotional responses in PD.

In the present study, we aimed to obtain a broader and more clinically anchored view of emotion processing in PD. We have included emotional stimuli from three different expression modalities -facial, bodily, and vocal expressions- equally balanced over positive and negative emotions. To our knowledge, body language has not been previously tested in an emotion recognition task with PD patients. Our objective is to take a multimodal approach to evaluate if there are group differences in emotion recognition, bias, and range. Additionally, we aim to relate emotion processing in PD and $\mathrm{HC}$ to clinical assessments of movement symptoms, individual cognitive status, executive control, attention, and working memory capacity.

Based on current PD literature we have four main hypotheses. We expect (a) that PD patients are less accurate than HC's at emotion recognition; and (b) that PD patients display an emotional bias in the form of enhanced valence and arousal scores for both positive and negative emotional stimuli. Therefore, we expect (c) PD patients to exhibit a larger emotional range to emotion stimuli. Finally, we expect (d) to find a relationship between PD patients' emotion task performance and their clinical scores, so that disease-related changes in clinical assessments are correlated to disease-related changes in task performance.

\section{Method}

\section{Participants}

33 patients (18 males, 15 females) diagnosed with idiopathic PD according to the United Kingdom Brain Bank Criteria (Hughes, Daniel, Kilford, \& Lees, 1992), and referred from the 
Center for Neurology at Academic Specialist Center in Stockholm, Sweden, participated in this study. Patients were treated with monotherapy or combination therapy and Levodopa equivalent daily dose (LEDD) ratings were computed for each patient separately. $29 \mathrm{HC}$ group participants (16 males, 13 females) were recruited for this study. Exclusion criteria for HC included diagnosis of any neurological or psychiatric disorder, treatment with anxiolytic medications or benzodiazepines, and claustrophobia. Means (M) and standard deviations (SD) of demographic and clinical data of PD patients and $\mathrm{HC}$ are given in Table 1 . The study was approved by the local ethics committee (DNR 2019-00542) and all participants gave informed consent, according to the Declaration of Helsinki, prior to involvement in the study.

Table 1

Participant Characteristics by Group

\begin{tabular}{lccc}
\hline \multicolumn{1}{c}{ Measure } & Healthy Controls & PD Patients & \\
& $M(S D)$ & $M(S D)$ & $p$ value \\
\hline Age in years & $64.4(8.6)$ & $63.5(9.5)$ & .680 \\
Response time per question $(s)$ & $43.2(13.1)$ & $40.7(10.9)$ & .341 \\
MoCA & $25.8(2.1)$ & $26.7(2.5)$ & .119 \\
FAB & $16.7(1.6)$ & $16.4(1.7)$ & .397 \\
BDI & $3.7(4.5)$ & $8.8(5.6)$ & $.000 *$ \\
LEDD & N/A & $545.6(307.6)$ & N/A \\
MDS-UPDRS-III & N/A & $17.3(10.5)$ & N/A \\
Modified H\&Y & N/A & $1.58(0.8)$ & N/A \\
\hline
\end{tabular}

Note. Response time refers to average amount of time taken to answer each question on the Emotion Survey; $s=$ seconds $; \mathrm{PD}=$ Parkinson's disease $; \mathrm{MoCA}=$ The Montreal Cognitive Assessment; FAB = Frontal Assessment Battery (Dubois et al., 2000); BDI = Beck’s Depression Inventory (Beck et al., 1974); LEDD = Levodopa 
Equivalent Daily Dose rating (Tomlinson et al., 2010); MDS-UPDRS-III = Movement Disorders Society Unified Parkinson's Disease Rating Scale, Part III (Goetz et al., 2008); Modified H\&Y = Modified Hoehn and Yahr staging scale (Goetz et al., 2004); N/A = Not Applicable; * indicates statistically significant results $(\mathrm{p}<.05)$.

\section{Emotion Stimuli}

The Emotion Survey consisted of short video or sound clips from the EU-emotion stimulus set (Lassalle et al., 2019; O'Reilly et al., 2016) showing actors portraying positive and negative emotions using three modalities - facial expressions (Face), body language (Body), or sound clips (Voice). Positive emotions included happiness, pride, joking, interest, excitement, and surprise. Negative emotions consisted of disgust, shame, fear, sadness, hurt, anger, worry, frustration, and boredom (example in Figure 1).

\section{Figure 1}

\section{Examples of Emotion Stimuli in The Emotion Survey}
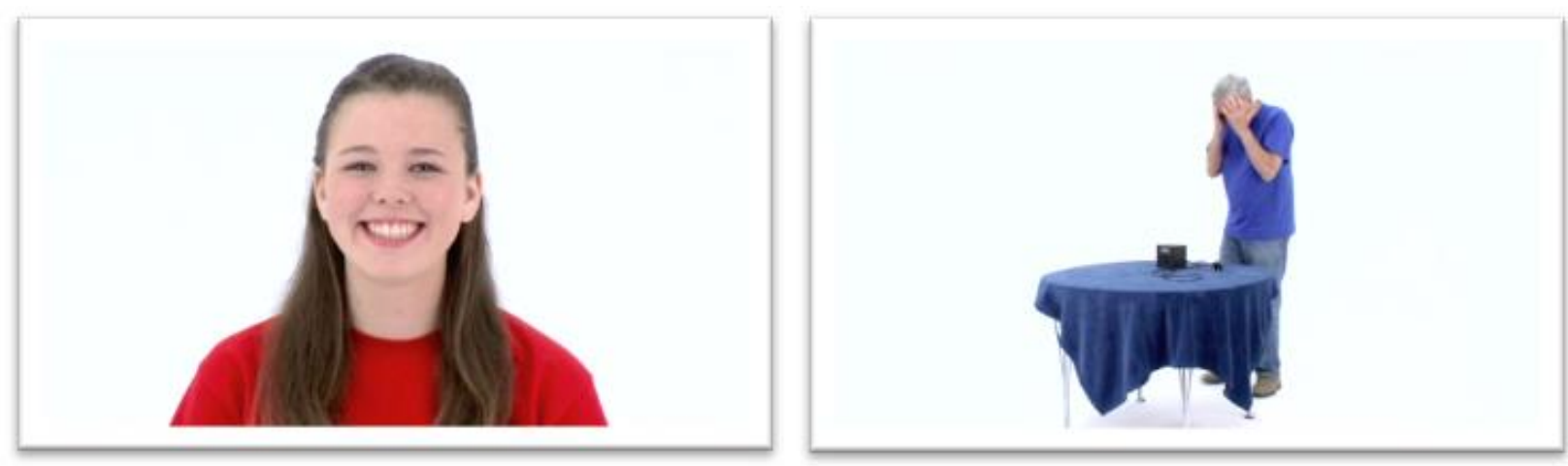

Note. Left: Actor portrayal for Face modality and 'Happiness' emotion. Right: Actor portrayal for Body modality and 'Frustrated' emotion.

The Emotion Survey consisted of 46 videos portraying different emotions via one of the three modalities: Face, Body, or Voice. Participants watched or listened to an emotion clip 
(Figure 1), then answered subscales related to (a) correct identification of the emotion portrayed, and (b) ratings of valence and arousal in response to the emotion. In the emotion identification subscales, participants were presented with five answer choices made up of the correct emotion plus four alternatives. Incorrect emotion answer choices had been validated by prior studies (Lassalle et al., 2019; O'Reilly et al., 2016) so that all answer choices were equally varied in their similarity or difference to the correct answer option in each emotion identification question. Participants then rated each emotion stimuli's subjective valence and arousal scores on a scale from one to five. When rating valence, the participants were asked "How do you perceive this emotional expression?" $(1=$ Very negative; $3=$ Neutral; $5=$ Very positive). When rating arousal, the participants were asked "How strongly do you react to this emotional expression?" (1 = Not at all, 3 = A little; 5 = Very strongly $)$.

\section{Clinical measures}

All participants completed three validated clinical measures. These consisted of (a) the Montreal Cognitive Assessment (MoCA, Gill et al., 2008) a screening tool for mild cognitive impairment (Gill et al., 2008); (b) The Frontal Assessment Battery, a short test to evaluate frontal lobe function and executive control (Dubois et al., 2000), and (c) Beck's Depression Inventory (BDI), a self-report measure of severity of depression (Beck et al., 1974).

LEDD ratings were computed by adding together the standardized LEDD rating for each antiparkinsonian drug taken by each patient included in the study (Tomlinson et al., 2010). LEDD ratings were calculated by P.T. and P.S., neurologists from Karolinska Hospital's Center for Neurology. PD patients completed two additional evaluations aimed at determining general clinical function related to their PD diagnosis. These included the Movement Disorders 
Society-Unified Parkinson's Disease Rating Scale, Part III (MDS-UPDRS-III, (Goetz et al., 2008), and the Modified Hoehn \& Yahr (H\&Y) staging scale (Goetz et al., 2004).

\section{Data Preparation and Statistical Analysis}

Gender was collapsed throughout all tasks because sex differences in emotional processing were not within the scope of this study. Statistical analysis was conducted using Statistica (TIBCO, 13.5.0).

\section{Emotion Recognition Analysis}

Correct responses on the emotion recognition task yielded one point per question (for 46 total points) while incorrect responses yielded no points. Student's t-tests were conducted to determine if there were group differences in emotion recognition between PD patients and HC.

\section{Bias and Range Analyses}

To prepare data for analysis of the specific hypotheses regarding bias and range, all stimuli used in the emotion survey were first categorized as either positive or negative emotions, and neutral stimulus items were excluded from the analysis. To calculate the composite measures for the bias analysis, the scores for all stimuli were pooled into a positive category, a negative category and an "all" category (pooling all stimuli from both categories into one), separately for each measure (valence, arousal, recognition) and stimulus modality (face, body, voice). Range measures were then computed by subtracting the score of the negative category from that of the positive category, separately for each measure and stimulus modality. Separate sets of $3 \times 2$ between-group analyses of variances (ANOVAs) were then conducted for each measure, using one set of analyses to address the bias hypothesis, and another set of analyses to address the range hypothesis.

\section{Clinical Relationship Analysis}


A correlation matrix was conducted between all clinical measures to assess the relationships among the scales. All participants' emotion recognition scores, as well as their positive, negative, average and range measures of arousal and valence in each emotion modality, were correlated with clinical measures using Pearson's correlation coefficient $r$. Within-group differences in responses to the emotion survey were also correlated to PD patient's LEDD ratings and clinical scores on the MDS-UPDRS-III, and H \& Y staging scale. Statistical significance for all analyses was set to $\mathrm{p}<.05$.

\section{Results}

\section{Emotion Recognition}

Analysis comparing the average positive, negative, and range emotion recognition scores between groups revealed group differences between average emotion recognition score $(\mathrm{p}=$ $.028)$ and average positive emotion recognition score $(p=.037)$. In both instances, the mean score for the PD patient group was higher than the mean $\mathrm{HC}$ group score, thereby indicating more accurate emotion recognition in the PD patient group (Table 2).

\section{Table 2}

Student's T-test for Group Differences in Emotion Recognition Accuracy.

\begin{tabular}{cccc}
\hline Recognition & Healthy Controls & PD Patients & $p$ value \\
accuracy & Mean \% (SD) & Mean \% (SD) & \\
\hline Average & $77 \%(.06)$ & $80 \%(05)$ & $.028^{*}$ \\
Negative & $79 \%(.63)$ & $82 \%(.06)$ & .169 \\
Positive & $74 \%(.09)$ & $78 \%(.06)$ & $.037^{*}$ \\
Range & $-5 \%(.09)$ & $-3 \%(.07)$ & .356 \\
\hline
\end{tabular}

Note. $*$ indicates $p$ value with significance level $<.05$ 


\section{Bias and Range}

Results of the separate $3 \times 2$ between-group ANOVAs for the composite "all" measures of valence, arousal, and response accuracy revealed no main effects of group on bias in valence or arousal. Similarly, when 3 x 2 between-group ANOVAs were conducted for valence and arousal range, no main effects of group were found.

\section{Relationships between clinical scales}

Table 3 shows the correlation matrix conducted to explore relationships among clinical measures MoCA, FAB, BDI, MDS-UPDRS-III and H\&Y. There were no significant correlations between these measures in the HC group, therefore correlations for the PD patient group only are reported.

Table 3

Correlations Among Clinical Scores in the PD Patient Group

\begin{tabular}{|c|c|c|c|c|c|c|}
\hline & $\mathrm{MoCA}$ & FAB & BDI & LEDD & MDS-UPDRS-III & $\mathrm{H} \& \mathrm{Y}$ \\
\hline & $\mathrm{r}(p)$ & $\mathrm{r}(p)$ & $\mathrm{r}(p)$ & $\mathrm{r}(p)$ & $\mathrm{r}(p)$ & $\mathrm{r}(p)$ \\
\hline $\mathrm{MoCA}$ & 1 & $.62(.000)^{\mathrm{a}}$ & - & - & - & - \\
\hline FAB & $.62(.000)^{\mathrm{a}}$ & 1 & - & - & $-.39(.029)^{b}$ & - \\
\hline BDI & - & - & 1 & - & - & $.36(.048)^{\mathrm{c}}$ \\
\hline LEDD & & & & 1 & & \\
\hline MDS- & - & $-.39(.029)^{b}$ & - & - & 1 & $.79(.000)^{\mathrm{d}}$ \\
\hline UPDRS-III & & & & & & \\
\hline $\mathrm{H} \& \mathrm{Y}$ & - & - & $.36(.048)^{\mathrm{c}}$ & - & $.79(.000)^{\mathrm{d}}$ & 1 \\
\hline
\end{tabular}

Note. There were no significant correlations between clinical measures in the HC group. MoCA $=$ The Montreal Cognitive Assessment; FAB = Frontal Assessment Battery; BDI = Beck's Depression Inventory; LED = Levodopa Equivalent Dose rating; MDS-UPDRS-III = Unified Parkinson's Disease Rating Scale, Part III; H\&Y = Hoehn \& Yahr Staging Scale; $\mathrm{r}=$ Pearson's correlation coefficient $r ; p=\mathrm{p}$-value, significance level $<.05$. 
In more detail, the PD patient group results show:

3a. Decreased cognitive function (MoCA) is correlated with decreased frontal lobe function and executive control (FAB)

3b. Decreased frontal lobe function and executive control (FAB) are correlated with more pronounced symptoms of PD (MDS-UPDRS-III).

3c. More pronounced physical symptoms of PD (H\&Y) are correlated with greater severity of depression (BDI).

3d. The two clinical scales meant to measure progression of physical PD symptoms MDSUPDRS-III and H\&Y) are highly correlated.

\section{Clinical scores and emotion recognition}

There were no significant correlations between PD patients' clinical scores and average, positive, negative, and range emotion recognition scores.

\section{Clinical scores and bias}

Clinical scores were correlated with the average positive, negative, and total valence and arousal scores for each modality using Pearson's $r$ correlation coefficient. Values of all results are reported in Table 4, with corresponding detailed descriptions given in the text. Detailed descriptions are listed using lowercase letters and matching letters can be found in superscript next to the corresponding statistical value in Table 4. No significant correlations were found

for the HC group in the Clinical scores and bias analysis, so only significant PD patient results are reported in detail. 


\section{Table 4}

Significant Correlations Between Clinical Scores and Measures of Valence and Arousal for PD Patients

\begin{tabular}{|c|c|c|c|c|c|c|c|}
\hline \multicolumn{8}{|c|}{ Valence: "How positive or negative is this emotion to you?" } \\
\hline \multicolumn{2}{|c|}{ Emotion measure } & $\mathrm{MoCA}$ & FAB & BDI & LEDD & $\mathrm{H} \& \mathrm{Y}$ & MDS-UPDRS-III \\
\hline \multirow{4}{*}{ All } & POS & $-.44(.011)^{\mathrm{a}}$ & $-.42(.015)^{\mathrm{d}}$ & $.36(.042)^{\mathrm{g}}$ & - & - & - \\
\hline & NEG & - & $\frac{-1.017)}{-}$ & $-.40(.020)^{h}$ & $.36(.045)^{\mathrm{n}}$ & - & - \\
\hline & $\mathrm{AVG}$ & - & - & $\begin{array}{c}-.70(.020) \\
-\end{array}$ & - & - & - \\
\hline & RANGE & - & - & $.44(.010)^{\mathrm{u}}$ & - & - & - \\
\hline \multirow{4}{*}{ Body } & POS & - & $-.35(.046)^{\mathrm{e}}$ & $.37(.032)^{\mathrm{i}}$ & - & - & - \\
\hline & NEG & - & - & $-.35(.046)^{j}$ & - & - & - \\
\hline & AVG & - & - & - & - & - & - \\
\hline & RANGE & - & - & $.41(.017)^{\mathrm{v}}$ & - & - & - \\
\hline \multirow{4}{*}{ Face } & POS & - & $-.36(.042)^{f}$ & - & - & - & - \\
\hline & NEG & - & $\begin{array}{c}-.00(.0+2) \\
-\end{array}$ & $-.45(.008)^{\mathrm{k}}$ & $.41(.022)^{\circ}$ & - & - \\
\hline & AVG & - & - & - & - & - & - \\
\hline & RANGE & - & - & $.49(.004)^{w}$ & - & - & - \\
\hline \multirow{4}{*}{ Voice } & POS & $-.58(.000)^{b}$ & - & - & - & - & - \\
\hline & NEG & - & - & - & - & - & - \\
\hline & AVG & $-.35(.048)^{c}$ & - & - & - & - & - \\
\hline & RANGE & $-.38(.027)^{\mathrm{p}}$ & - & - & - & - & - \\
\hline
\end{tabular}

\begin{tabular}{|c|c|c|c|c|c|c|c|}
\hline \multicolumn{8}{|c|}{ Arousal: "How strongly do you react to this emotional expression?" } \\
\hline \multicolumn{2}{|c|}{ Emotion measure } & MoCA & FAB & BDI & LEDD & $\mathrm{H} \& \mathrm{Y}$ & MDS-UPDRS-III \\
\hline \multirow{4}{*}{ All } & POS & - & - & - & - & - & - \\
\hline & NEG & - & - & - & - & - & - \\
\hline & AVG & - & - & - & - & - & - \\
\hline & RANGE & $-.43(.012)^{\mathrm{q}}$ & $-.40(.021)^{\mathrm{r}}$ & - & - & - & - \\
\hline \multirow{4}{*}{ Body } & POS & - & - & - & - & - & - \\
\hline & NEG & - & - & - & - & - & - \\
\hline & AVG & - & - & - & - & - & - \\
\hline & RANGE & $.48(.008)^{\dagger}$ & $-.35(.045)^{\mathrm{s}}$ & - & - & - & - \\
\hline \multirow{4}{*}{ Face } & POS & - & - & - & - & - & - \\
\hline & NEG & - & - & - & - & - & - \\
\hline & AVG & - & - & - & - & - & - \\
\hline & RANGE & - & $-.38(.028)^{\mathrm{t}}$ & - & - & - & - \\
\hline \multirow{4}{*}{ Voice } & POS & - & - & - & - & - & - \\
\hline & NEG & - & - & $.36(.039)^{1}$ & - & - & - \\
\hline & AVG & - & - & $.36(.038)^{\mathrm{m}}$ & - & - & - \\
\hline & RANGE & - & - & - & - & - & - \\
\hline
\end{tabular}

Note. POS = positive; $\mathrm{NEG}=$ negative $\mathrm{AVG}=$ average MoCA = The Montreal Cognitive Assessment; FAB = Frontal Assessment Battery; $\mathrm{BDI}=$ Beck's Depression Inventory; $r=$ Pearson's correlation coefficient $r ; *=\mathrm{p}$-value $\leq .05 ; \dagger=$ correlation in HC group. 
MoCA scores and emotional bias. Increased cognitive impairment (lower MoCA score) was significantly correlated with:

4a. More positive ratings of positive emotional stimuli across all modalities

4b. More positive ratings of positive emotional stimuli in the Voice modality

4c. Average increase in ratings of emotional stimuli in the Voice modality

FAB scores and emotional bias. Decreased frontal lobe function and executive control (lower FAB score) were significantly correlated with:

4d. More positive ratings of positive emotional stimuli across all modalities

4e. More positive ratings of positive emotional stimuli in the Body modality

4f. More positive ratings of positive emotional stimuli in the Face modality

BDI scores and emotional bias. Greater severity of depression (higher BDI score) was significantly correlated with:

4g. More positive ratings of positive emotional stimuli across all modalities

4h. More negative ratings of negative emotional stimuli across all modalities

4i. More positive ratings of positive emotional stimuli in the Body modality

4j. More negative ratings of negative emotional stimuli in the Body modality

4k. More negative ratings of negative emotional stimuli in the Face modality

41. Stronger reactions to negative emotional stimuli in the Voice modality

4m. Average increase in reactions to emotional stimuli in the Voice modality

LEDD scores for PD patients and emotional bias. An increased daily dose of antiparkinsonian medication (higher LEDD scores) was significantly correlated with:

4n. More positive ratings of negative emotional stimuli

4o. More positive ratings of negative emotional stimuli in the Face modality 


\section{Clinical scores and range}

Clinical scores were correlated with emotional range scores for each modality using Pearson's $r$ correlation coefficient. All results are reported in Table 4, with detailed descriptions reported below. Detailed descriptions are listed using lowercase letters and matching letters can be found in superscript next to the corresponding statistical value in Table 4. The HC group had only one significant correlation in the range category which has been reported in Table $4 \mathrm{~b}$. All other significant correlations between clinical score and range were specific to the PD patient group.

MoCA scores and emotional range. Increased cognitive impairment (lower MoCA score) was significantly correlated with:

$4 p$. Larger range between ratings of positive and negative stimuli in the Voice modality (Figure 2a)

4q. Larger range in reactions to positive and negative emotional stimuli across all modalities (Figure 2b)

FAB scores and emotional range. Decreased frontal lobe function and executive control (lower FAB scores) were significantly correlated with:

4r. Larger range in reactions to positive and negative emotional stimuli across all modalities

4s. Larger range in reactions to positive and negative emotional stimuli in the Body modality (Figure 2c)

4t. Larger range in reactions to positive and negative emotional stimuli in the Face modality (Figure 2d)

BDI scores and emotional range. Greater severity of depression (higher BDI score) was significantly correlated with: 
$4 \mathrm{u}$. Larger range between ratings of positive and negative stimuli across modalities

4v. Larger range between ratings of positive and negative stimuli in the Body modality (Figure 2e)

4w. Larger range between ratings of positive and negative stimuli in the Face modality (Figure 2f)

\section{Figure $2(a-f)$}

Significant Correlations Among Emotional Ranges and Clinical Measures in the PD Patient Group
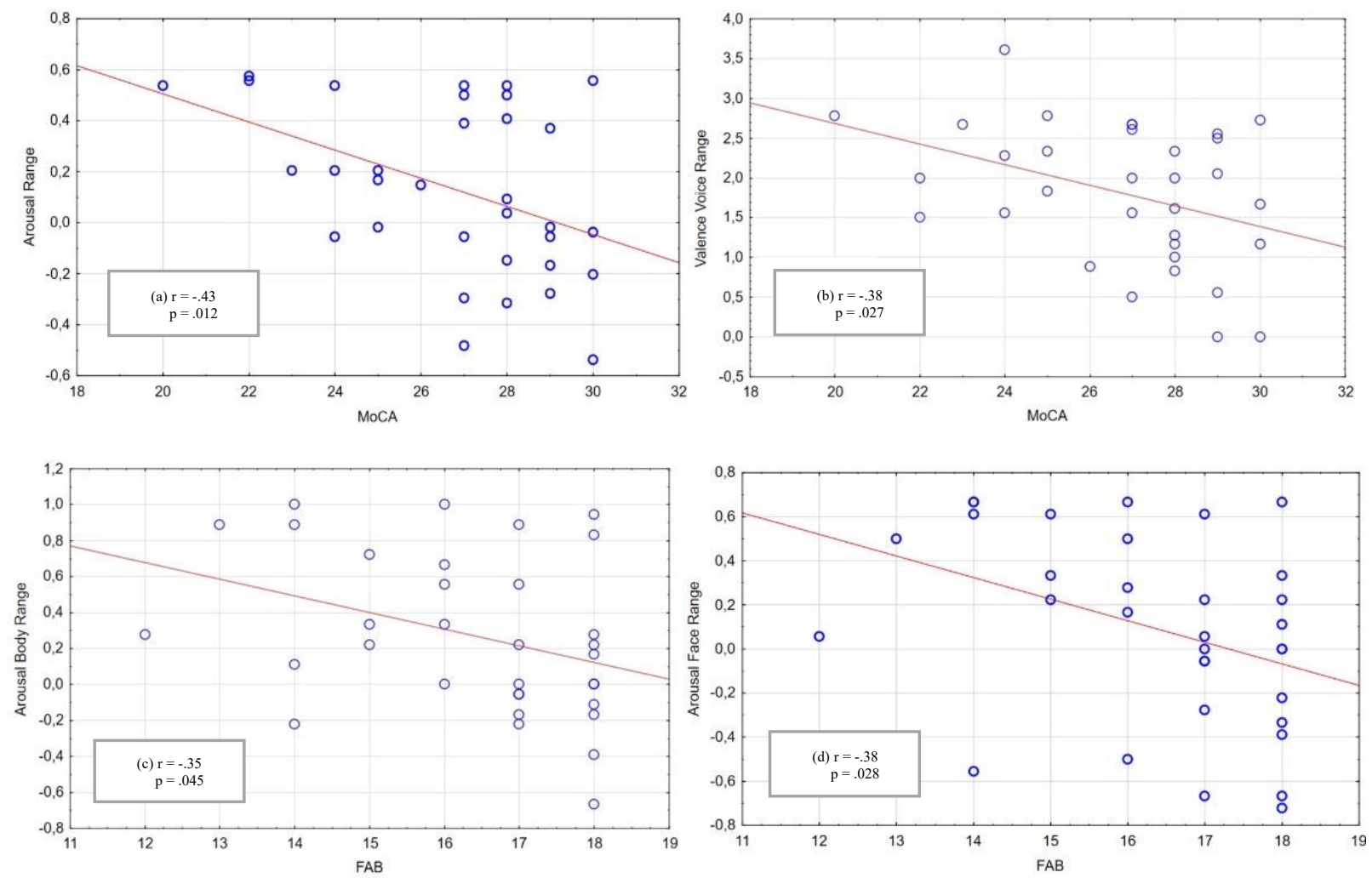

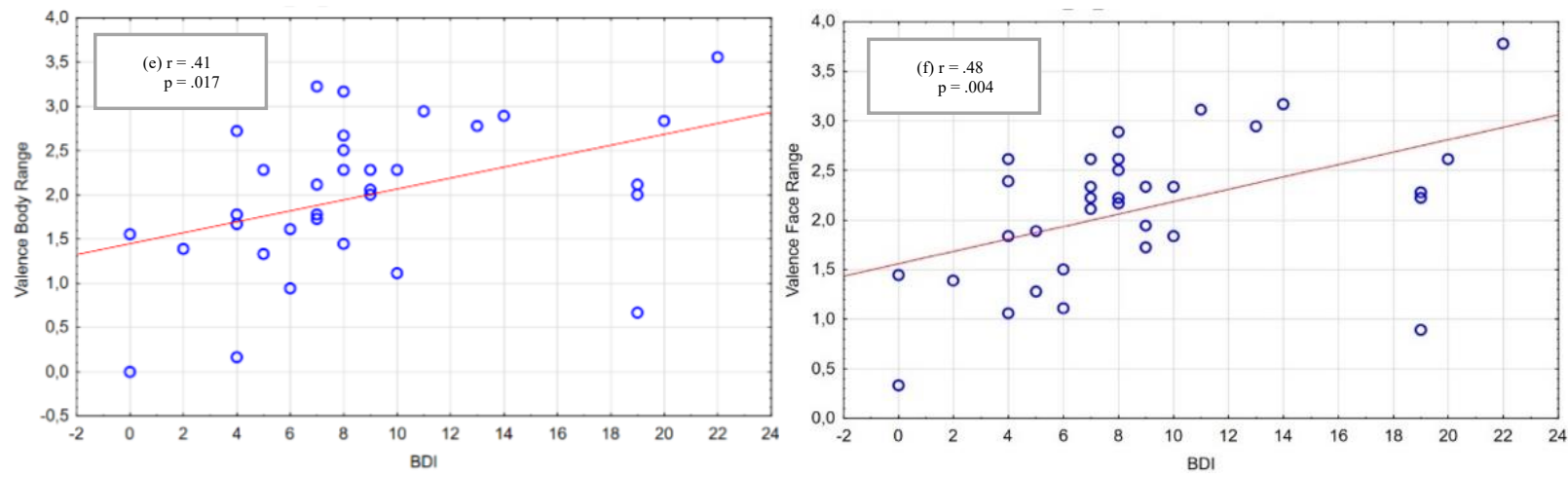

Note. Higher scores on MoCA and FAB indicate better cognitive function and executive control (Dubois, Slachevsky, Litvan, \& Pillon, 2000; Gill, Freshman, Blender, \& Ravina, 2008). Higher BDI scores reflect more serious symptoms of depression (Beck, Rial, \& Rickels, 1974). There was only one significant correlation between clinical scores and emotional range in the $\mathrm{HC}$ group (not shown).

\section{MDS-UPDRS-II and H\&Y analyses}

PD data was analyzed for within-group differences related to emotional perception and the MDS-UPDRS-III and H\&Y staging scale - both clinical measures specific to PD. However, the results of Pearson correlations between these clinical scales and measures of emotion recognition, arousal, valence, and range showed no within-group differences.

\section{Discussion}

The present study utilized the Emotion Survey to compare how PD patients and HC recognize, rate, and react to emotional stimuli presented in different modalities. Emotion stimuli were presented in Face, Body, or Voice modalities and data related to emotional recognition, bias, and range was collected and then correlated with clinical outcome measures that assess individual cognitive status, executive control, attention and working memory. The main results based on our previous hypotheses are presented below.

\section{Emotion recognition}

We compared the PD patient group and the HC group on emotion recognition accuracy and found significant differences between the groups. However, in contradiction to our earlier 
hypothesis, PD patients were more accurate (3-4\% better) than $\mathrm{HC}$ across modalities and in response to both positive and negative emotion stimuli. This result is particularly compelling in light of the intense focus of previous research on emotion identification tasks as the measure of emotional impairment in PD patients.

\section{Bias and Range}

There were no main effect group differences between the PD patients and HC Bias and Range scores. We had initially hypothesized that there would be differences in both of these categories, but since PD patients included in this study were in the early stages of disease progression $(\mathrm{H} \& \mathrm{Y}$ mean score $=1.58)$, the differences between groups were not large enough for group's total scores to differ significantly. Future studies could examine these hypotheses again by recruiting PD patients more advanced in disease progression.

\section{Clinical relationships}

We hypothesized there would be a relationship between PD patients' emotion task performance and their clinical scores, so that disease-related changes in clinical assessments would be correlated to changes in task performance. We believe the data confirms this hypothesis for several reasons. The clinical tests implemented in this study were chosen with the consideration that they might best reflect aspects of early stage PD. A correlation matrix amongst the clinical measures confirmed their interconnectedness, with PD-specific measures (MDS-UPDRS-III, H\&Y) highly correlating with each other as well as with other general clinical tests (MoCA, FAB, BDI). These tests reflect aspects of PD progression. In support of this theory, the results showed that significant correlations between emotion stimuli data and clinical scores were, with only one exception, exclusive to the PD patient group. 
In the bias, range, and clinical scores analyses, the results indicated a pattern of significant correlations between clinical scores and how PD patients rated (valence) and reacted (arousal) to emotional stimuli. PD patients with greater cognitive impairment and decreased frontal lobe function and executive control (i.e. lower MoCA and FAB scores) consistently gave higher positive ratings to positive emotional stimuli. PD patients with more severe depressive symptoms showed a similar pattern in their ratings of positive emotional stimuli, while also giving lower ratings to negative emotional stimuli. Whereas previous research more heavily focused on negative emotional stimuli has shown a negativity bias in PD patients, the current study suggests that PD patients actually are more reactive to both positive and negative emotional stimuli. PD patients, therefore, exhibited a greater emotional range in connection to clinical scores than HC. Clinical scores meant to reflect cognitive decline, depression, and disease progression correlated with higher variability in emotional ranges to emotional stimuli across modality. While no main group differences were found between the three emotion modalities, taking a multimodal approach was still beneficial in finding relationships between clinical measures and responses to emotion stimuli. Correlations between clinical scores and emotional responses to Face and Body modalities tended to reveal similar patterns in the data, with responses to the Face modality, in particular, proving to be somewhat redundant to responses in the Body modality. Correlations between clinical tests and emotional responses to the Voice modality, however, exposed relationships between the emotion data and clinical scores that were unique to this modality.

The overall pattern of results indicate that PD patients experience a generalized increase in perception of both positive and negative emotions which could serve as an indicator of altered emotional processing related to early PD. This increase is seen both in terms of 
emotional responses to stimuli, and also in emotion recognition accuracy. The loss of dopaminergic neurons characteristic of PD may help to explain changes in emotional regulation. Abraham and colleagues (2014) described the multifaceted role of dopamine well when writing that, "dopamine is involved in the circumstances that produce learning (e.g., prediction error, coding of stimulus salience); it mediates the content of that learning (e.g., hedonic value of associations); and it modulates the expression of learning in performance (e.g., response vigor, memory retrieval)" (p. 65). Alterations in dopaminergic activity may lead to dysregulation in brain regions, such as the ventral striatum and basolateral amygdala, which are reliant on dopaminergic input to properly code salience of emotional stimuli and regulate emotional response to emotional stimuli (Fadok, Darvas, Dickerson, \& Palmiter, 2010; Fadok, Dickerson, \& Palmiter, 2009).

\section{Limitations}

While our study suggests differences in aspects related to emotional processing in PD patients, there are two potentially confounding factors - BDI scores and dopaminergic therapy. BDI scores differed significantly between groups and it is, therefore, impossible for us to rule out that depressive symptoms may be responsible for any group differences. We have chosen to view BDI as one measure or component which, together with other clinical measures, could indicate disease progression. It should be noted that some points in BDI relates to symptoms common in PD (e.g. fatigue, sleep problems, appetite, concentration difficulties) and may not only reflect a depressed state. Indeed, BDI scores were found to be significantly correlated with H\&Y staging scale scores, a more direct measure of disease progression. Another limitation of this study is that all PD patients were on levodopa or taking dopamine agonists which have been associated with a masking of emotional impairment in previous studies (Lundqvist et al., 
2017). Our results support these previous findings in showing that PD patients taking higher daily doses of antiparkinsonian medications (as indicated by increased LEDD scores) were significantly more likely to rate negative emotional stimuli as less negative than patients on lower daily doses of antiparkinsonian medication. Since the current study has primarily found an increase in emotional ranges in conjunction with clinical scores in PD patients, it is more likely that our results would have been more robust if patients in an off-medication condition were included. Still, future studies could compare these three modalities with PD patients in an off-medication condition, as well as control for differences in BDI scores.

\section{Conclusion}

Our findings show the important role of emotional range as a detector of emotion processing changes in early PD. Correlations in all modalities between emotional bias, range, and clinical scores for PD patients suggests that emotion regulation, instead of emotion recognition, could be a more sensitive indicator of alterations in emotional processing early in disease progression. 


\section{References}

Aarsland, D., Påhlhagen, S., Ballard, C. G., Ehrt, U., \& Svenningsson, P. (2011). Depression in Parkinson disease--epidemiology, mechanisms and management. Nat Rev Neurol, 8(1), 35-47. doi:10.1038/nrneurol.2011.189

Abraham, A. D., Neve, K. A., \& Lattal, K. M. (2014). Dopamine and extinction: a convergence of theory with fear and reward circuitry. Neurobiol Learn Mem, 108, 65-77. doi:10.1016/j.nlm.2013.11.007

Beck, A. T., Rial, W. Y., \& Rickels, K. (1974). Short form of depression inventory: crossvalidation. Psychol Rep, 34(3), 1184-1186.

Blonder, L. X., Slevin, J. T., Kryscio, R. J., Martin, C. A., Andersen, A. H., Smith, C. D., \& Schmitt, F. A. (2013). Dopaminergic modulation of memory and affective processing in Parkinson depression. Psychiatry Res, 210(1), 146-149. doi:10.1016/j.psychres.2013.06.003

Breitenstein, C., Van Lancker, D., Daum, I., \& Waters, C. H. (2001). Impaired perception of vocal emotions in Parkinson's disease: influence of speech time processing and executive functioning. Brain Cogn, 45(2), 277-314. doi:10.1006/brcg.2000.1246

De Risi, M., Di Gennaro, G., Picardi, A., Casciato, S., Grammaldo, L. G., D'Aniello, A., . . Modugno, N. (2018). Facial emotion decoding in patients with Parkinson's disease. Int J Neurosci, 128(1), 71-78. doi:10.1080/00207454.2017.1366475

Dubois, B., Slachevsky, A., Litvan, I., \& Pillon, B. (2000). The FAB: a Frontal Assessment Battery at bedside. Neurology, 55(11), 1621-1626. doi:10.1212/wnl.55.11.1621 
Dujardin, K., Blairy, S., Defebvre, L., Duhem, S., Noël, Y., Hess, U., \& Destée, A. (2004). Deficits in decoding emotional facial expressions in Parkinson's disease. Neuropsychologia, 42(2), 239-250. doi:10.1016/s0028-3932(03)00154-4

Fadok, J. P., Darvas, M., Dickerson, T. M., \& Palmiter, R. D. (2010). Long-term memory for pavlovian fear conditioning requires dopamine in the nucleus accumbens and basolateral amygdala. PLoS One, 5(9), e12751. doi:10.1371/journal.pone.0012751

Fadok, J. P., Dickerson, T. M., \& Palmiter, R. D. (2009). Dopamine is necessary for cuedependent fear conditioning. J Neurosci, 29(36), 11089-11097.

doi:10.1523/JNEUROSCI.1616-09.2009

Gill, D. J., Freshman, A., Blender, J. A., \& Ravina, B. (2008). The Montreal cognitive assessment as a screening tool for cognitive impairment in Parkinson's disease. Mov Disord, 23(7), 1043-1046. doi:10.1002/mds.22017

Goetz, C. G., Poewe, W., Rascol, O., Sampaio, C., Stebbins, G. T., Counsell, C., .. Disease, M. D. S. T. F. o. R. S. f. P. s. (2004). Movement Disorder Society Task Force report on the Hoehn and Yahr staging scale: status and recommendations. Mov Disord, 19(9), 10201028. doi:10.1002/mds.20213

Goetz, C. G., Tilley, B. C., Shaftman, S. R., Stebbins, G. T., Fahn, S., Martinez-Martin, P., .. . Force, M. D. S. U. R. T. (2008). Movement Disorder Society-sponsored revision of the Unified Parkinson's Disease Rating Scale (MDS-UPDRS): scale presentation and clinimetric testing results. Mov Disord, 23(15), 2129-2170. doi:10.1002/mds.22340

Gray, H. M., \& Tickle-Degnen, L. (2010). A meta-analysis of performance on emotion recognition tasks in Parkinson's disease. Neuropsychology, 24(2), 176-191. doi:10.1037/a0018104 
Hughes, A. J., Daniel, S. E., Kilford, L., \& Lees, A. J. (1992). Accuracy of clinical diagnosis of idiopathic Parkinson's disease: a clinico-pathological study of 100 cases. J Neurol Neurosurg Psychiatry, 55(3), 181-184. doi:10.1136/jnnp.55.3.181

Hälbig, T. D., Assuras, S., Creighton, J., Borod, J. C., Tse, W., Frisina, P. G., . . Olanow, C. W. (2011). Differential role of dopamine in emotional attention and memory: evidence from Parkinson's disease. Mov Disord, 26(9), 1677-1683. doi:10.1002/mds.23728

Ille, R., Wabnegger, A., Schwingenschuh, P., Katschnig-Winter, P., Kögl-Wallner, M., Wenzel, K., \& Schienle, A. (2016). Intact emotion recognition and experience but dysfunctional emotion regulation in idiopathic Parkinson's disease. J Neurol Sci, 361, 72-78. doi:10.1016/j.jns.2015.12.007

Kalia, L. V., \& Lang, A. E. (2015). Parkinson's disease. Lancet, 386(9996), 896-912. doi:10.1016/S0140-6736(14)61393-3

Kan, Y., Kawamura, M., Hasegawa, Y., Mochizuki, S., \& Nakamura, K. (2002). Recognition of emotion from facial, prosodic and written verbal stimuli in Parkinson's disease. Cortex, 38(4), 623-630. doi:10.1016/s0010-9452(08)70026-1

Lassalle, A., Pigat, D., O'Reilly, H., Berggen, S., Fridenson-Hayo, S., Tal, S., . . Lundqvist, D. (2019). The EU-Emotion Voice Database. Behav Res Methods, 51(2), 493-506. doi:10.3758/s13428-018-1048-1

Lundqvist, D., Svärd, J., Michelgård Palmquist, Å., Fischer, H., \& Svenningsson, P. (2017). Patients with Parkinson's disease display a dopamine therapy related negative bias and an enlarged range in emotional responses to facial emotional stimuli. Neuropsychology, 31(6), 605-612. doi:10.1037/neu0000371 
O'Reilly, H., Pigat, D., Fridenson, S., Berggren, S., Tal, S., Golan, O., . . . Lundqvist, D. (2016). The EU-Emotion Stimulus Set: A validation study. Behav Res Methods, 48(2), 567-576. doi:10.3758/s13428-015-0601-4

Pell, M. D., \& Leonard, C. L. (2005). Facial expression decoding in early Parkinson's disease. Brain Res Cogn Brain Res, 23(2-3), 327-340. doi:10.1016/j.cogbrainres.2004.11.004

Postuma, R. B., Berg, D., Stern, M., Poewe, W., Olanow, C. W., Oertel, W., . . Deuschl, G. (2015). MDS clinical diagnostic criteria for Parkinson's disease. Mov Disord, 30(12), 1591-1601. doi:10.1002/mds.26424

Péron, J., Dondaine, T., Le Jeune, F., Grandjean, D., \& Vérin, M. (2012). Emotional processing in Parkinson's disease: a systematic review. Mov Disord, 27(2), 186-199. doi:10.1002/mds. 24025

Schapira, A. H. V., Chaudhuri, K. R., \& Jenner, P. (2017). Non-motor features of Parkinson disease. Nat Rev Neurosci, 18(7), 435-450. doi:10.1038/nrn.2017.62

Tomlinson, C. L., Stowe, R., Patel, S., Rick, C., Gray, R., \& Clarke, C. E. (2010). Systematic review of levodopa dose equivalency reporting in Parkinson's disease. Mov Disord, 25(15), 2649-2653. doi:10.1002/mds.23429

Wabnegger, A., Ille, R., Schwingenschuh, P., Katschnig-Winter, P., Kögl-Wallner, M., Wenzel, K., \& Schienle, A. (2015). Facial Emotion Recognition in Parkinson's Disease: An fMRI Investigation. PLoS One, 10(8), e0136110. doi:10.1371/journal.pone.0136110

Yip, J. T., Lee, T. M., Ho, S. L., Tsang, K. L., \& Li, L. S. (2003). Emotion recognition in patients with idiopathic Parkinson's disease. Mov Disord, 18(10), 1115-1122. doi:10.1002/mds.10497 
